noch Sonnenlicht reflektieren, verschiedene Schichtungen vorhanden sind, welche für unsere Breiten entsprechende Lichtabstufungen zur Folge haben. So kommen wir zu dem Schlusse, daß unsere Wasserstoffatmosphäre nicht von einer, sondern mindestens von zwei Gasarten iberlagert sei, die in ihren Schichtungsgrenzen ohne Zweifel gegenseitig gemischt sind.

Wir werden nun leicht auf den Gedanken geleitet, daß auch der innere Kegel des Zodiakallichts auf eine derartige Schichtungsgrenze hindeute. Ob dies zutrifft, oder ob dieses Anschwellen der Lichtintensitát gegen den Horizont als die einfache Folge der stark zunehmenden Tiefe und Dichtigkeit der reflektierenden Atmosphärenschichten aufzufassen sei, kann nicht sofort beantwortet werden. Schon die der Erde am nächsten stehenden reflektierenden Iuftschichten des Zodiakallichts weisen uns auf einen Erdabstand von mindestens $200 \mathrm{~km}$, wo nach Wegener die Übergangszone des Wasserstoffs zun Geokoronium liegt. Wenn nun dieser innere Kern wirklich eine besondere Schichtung der Atmosphäre verraten sollte, so stehen wir vor der schwierigen Untersuchung, ob für unsere Beobachtung die Wasserstoffschicht in die Geokoroniumsphäre hinaufragt, oder ob wir es mit einer besonderen Gasschicht zu tun haben. Im ersten Falle müßten wir die obere Grenze des Wasserstoffs in bedeutend höhere Regionen verlegen, als man bis heute angenommen hat, und im zweiten Falle wäre die Wasserstoffatmosphäre nicht von $z$ wei, sondern von drei nachweisbaren Gasschichten überlagert.

Wir werden nun leicht auf den Gedanken geleitet, aus der Höhe der verschiedenen Dämmerungsstufen und dem jeweiligen Sonnenstande unter dem Horizonte die Höhe der verschiedenen Atmosphärenschichten abzuleiten. Gewiß geben uns derartige Untersuchungen wertvolle Schlüsse über den Bau unserer Lufthülle, und dennoch dürfte es gewagt sein,

Oberhelfenswil, Kt. St. Gallen, I 9 I 2 Juni. namentlich aus den äußersten lichtschwachen Grenzen die wirkliche Höhe der Atmosphäre direkt ableiten zu wollen. Wir haben mit einer schwerwiegenden Störung zu rechnen, welche namentlich die Resultate der letzten I)ämmerungsgrenze bedeutend herabsetzen wird. Es ist dies die Absorptionskraft der Atmosphäre zwischen der unteren reflektierenden Grenze bis zum Beobachter, die wir auf die äußerste und schwächste Dämmerungszone kaum richtig einschätzen können. Jedenfalls werden wir für unsere Erdatmosphäre zu Dimensionen gelangen, welche von den bisherigen Annahmen vollständig abweichen. Man hat sich bisher gewöhnlich begnügt, aus der Abnahme des Luftdruckes und aus der Fntzündungsgrenze der Meteore die wirkliche Höhe der Atmosphäre direkt abzuleiten. Doch diese beiden Grundlagen führen zu falschen Schlüssen. Es ist die Annahme wohl kaum anfechtbar, daß die letzten Zonen der Atmosphäre sehr allmählich in den Ather übergehen. Die in die Erdatmosphäre eintretenden Meteoriten werden daher erst dann in den Glühzustand versetzt, nachdem sie einen weiten Weg in der Erdatmosphäre zurückgelegt haben und in die tieferen Schichten derselben eingedrungen sind. Auch die Abnahme des Iuftdruckes gegen außen bietet kaum genügende Anhaltspunkte zur Berechnung des I uftmantels, da dem Eigengewicht der Atmosphäre die durch die Erdrotation bewirkte Zentrifugalkraft entgegenarbeitet. Wollen wir aber für die einzelnen Atmosphärenhöhen die Cegenwirkung der Zentrifugalkraft bestimmen, so begegnen wir einer weiteren erheblichen Schwierigkeit, indem die Winkelgeschwindigkeit der Erdrotation mit größter Wahrscheinlichkeit mit zunehmender Höhe in der Atmosphäre abnimmt. So steht die Meteorologie vor neuen, wichtigen aber ebenso schwer lösbaren Aufgaben, und es ist in erster Linie heute wohl an der Zeit, die veraltete Annahme einer zirka $200 \mathrm{~km}$ hohen Erdatmosphäre endgültig aufzugeben.

\section{F. Schmid.}

\title{
Mikrometermessungen von Doppelsternen 1911-1912.
}

Von C. Luplau-Fanssen und E. Buch-Andersen.

Die vorliegende Messungsreihe ist hauptsächlich während der Wintermonate auf der hiesigen Urania-Sternwarte ausgeführt worden, und sie bildet eine Fortsetzung der früher erschienenen. An den Messungen haben die beiden Mitarbeiter der Sternwarte $C$. Luplau-Fanssen (L) und E. BuchAndersen (A) teilgenommen. Unser Arbeitsprogramm umfaßt sowohl Bahnpaare als Paare mit wahrscheinlicher Bahnbewegung und endlich eine Anzahl von Paaren, welche ihre gegenseitige Lage scheinbar nicht ändern. Die letzteren, die wir aus verschiedenen Gründen auf unser Arbeitsprogramm aufgenommen haben, sind aber stets bei weniger ruhiger Luft als die erstgenannten gemessen worden. Die Messungen werden vorläufig auf der Sternwarte fortgesetzt. Die unten angegebenen Sternörter sind alle annäherungsweise auf das Äquinoktium r 910.0 bezogen. Die erste Kolumne gibt den Zeitpunkt der Beobachtung an, die zweite den Beobachter, die dritte den Positionswinkel, die vierte die Distanz, die fünfte die Sternzeit, und endlich gibt die letzte die bei der Beobachtung angewandte Vergrößerung an. Unter jedem Stern sind die Mittel der Beobachtungen und unsere Bemerkungen über die Sterngröße und Farbe der Komponenten etc. zu finden. Die Gestirne sind nach gerader Aufsteigung geordnet, und vor dem Namen des Sternes haben wir seine Nummer in dem Burnhamschen Kataloge angegeben.
B GC 35. $\Sigma_{4}$. $\quad 0^{\mathrm{h}} 5^{\mathrm{m}} \cdot 3+7^{\circ} 57^{\prime}$. I9Ir.78I L $92.95 \quad 5^{\text {". }} 44 \quad \mathrm{I}^{\mathrm{h}} 469$

2789. $\Sigma 730 . \quad 5^{\text {h }} 26^{\mathrm{m}} \cdot 9+17^{\circ} 0^{\prime}$. $\begin{array}{lllllll}1912.022 & \text { L } & \text { I } 41.63 & 9.548 & 6.5 & 308\end{array}$ \begin{tabular}{llllll}
1912.027 & $\mathrm{~L}$ & I 41.30 & $9.66 \mathrm{I}$ & 7.2 & 308 \\
\hline
\end{tabular} 3185. $\Sigma 855 . \quad 6^{\mathrm{h}} 4^{\mathrm{m}} \cdot 2+2^{\circ} 3^{\prime}$. I 9 I 2.022 L 1 I $3.9029 .665 \quad 7^{\mathrm{h}} 308$ $\begin{array}{lllll}1912.025 \quad \mathrm{~L} & \text { II } 4.13 & 29.062 & 7 & 308\end{array}$ 1912.023 I, II $4.02 \quad 29.364$

3380. $2911.66^{\mathrm{h}} 22^{\mathrm{m}} \cdot 6+4^{\circ} 8^{\prime}$.

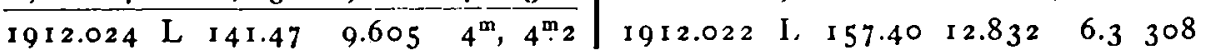

3402. I 919. AB. $6^{\mathrm{h}} 24^{\mathrm{m}} \cdot 5-6^{\mathrm{c}} 5^{8 \mathrm{t}}$. I 912.034 Lo $131.20 \quad 7^{\prime \prime} 410 \quad 6^{\text {h }} 3308$

3427. \ $921.6^{\mathrm{h}} 26^{\mathrm{m}} \mathrm{o}+11^{\circ} 19^{\prime}$. $\begin{array}{llllll}1912.037 & \mathrm{~L} & 5.88 & 15.932 & 6.7 & 308\end{array}$ 
4122. $\Sigma$ I I о. Castor. $7^{\mathrm{h}} 28^{\mathrm{m}} \cdot 6+32^{\circ} 5^{\prime}$.

I $912.024 \mathrm{~L} 219^{\circ} .30 \quad 5^{\text {. }} .289 \quad 5^{\text {h }} \cdot 7615$ $1912.027 \mathrm{~L} \quad 219.20 \quad 5.563 \quad 5.51000$ 1912.026 L $219.25 \quad 5.426$

4452. $\mathrm{\Sigma}_{1 \mathrm{I} 87 .} 8^{\mathrm{h}} 3^{\mathrm{m}} \cdot 8+3^{\circ} 27^{\prime}$. $\begin{array}{lllll}1912.024 \mathrm{~L} & 38.48 & 1.938 & 6 & 615\end{array}$ I912.027 L $39.13 \quad 1.983 \quad 6 \quad 615$ $1912.026 \mathrm{~L} \quad 38.80 \quad 1.96$ 1

5030. $\Sigma_{1338 .} 9^{\mathrm{h}} 5_{5^{\mathrm{m}}} \cdot 4+38^{\circ} 34^{\prime}$.

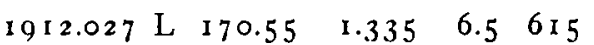

5388. $\Sigma_{1424}, \gamma$ Leonis. I $0^{\mathrm{h}} 14^{\mathrm{m}} \cdot 9+20^{\circ} 18^{\prime}$.

$\begin{array}{llllll}\text { I } 912.249 & \mathrm{~A} & \text { I I } 8.88 & 3.495 & 10.7 & 469\end{array}$ I912.307 A I I6.17 3.731 I $1.6 \quad 378$. I $912.278 \mathrm{~A}$ II $7.53 \quad 3.613$

5448. У $1439 . \quad 10^{\mathrm{h}} 25^{\mathrm{m} ! \mathrm{I}}+2 \mathrm{I}^{\circ} 16^{\prime}$.

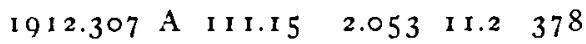
5944. - $\Sigma_{\text {I } 575 . ~}$ I I $^{\mathrm{h}} 47^{\mathrm{m}} \cdot 3+9^{\circ} 20^{\prime}$. $\begin{array}{llllll}1912.249 & \mathrm{~L} & 209.88 & 30.683 & 10.8 & 308\end{array}$ $\begin{array}{ll}1912.249 \text { A } 209.68 \quad 30.528 \text { I } 1.0 & 308\end{array}$ $7^{\mathrm{m}}, 8^{\mathrm{m}}$.

5968. $\Sigma_{1582 .} I_{1}{ }^{\mathrm{h}} 5 \mathrm{I}^{\mathrm{m}} \cdot 4+22^{\circ} 29^{\prime}$. $\begin{array}{lllll}\text { 1912.309 L } & 74.1 & \text { I } 1.638 & \text { I } 3.5 & 378\end{array}$

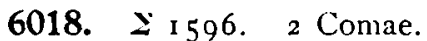
I $\mathrm{I}^{\mathrm{h}} 59^{\mathrm{m}} \cdot 6+2 \mathrm{I}^{\circ} 58^{\prime}$.

$\begin{array}{lllllll}1912.249 & \text { L } & 235.51 & 3.731 & \text { I I.8 } & 469\end{array}$ I 9 2.249 A $236.76 \quad 3.809$ r 1.5469 $\begin{array}{lllllll}1912.293 & \mathrm{~L} & 232.62 & 3.87 & \text { I } & 2.0 & 378\end{array}$ $\begin{array}{llllll}1912.293 \mathrm{~A} & 23.3 .90 & 4.006 & 12.1 & 378\end{array}$

I 912.271 L $234.06 \quad 3.801$

$1912.271 \mathrm{~A} 235.33 \quad 3.907$

6053. $\Sigma_{1606 . ~} 12^{\mathrm{h}} 6^{\mathrm{m}} \cdot 2+40^{\circ} 24^{\prime}$. $\begin{array}{lllll}\text { I9I2.203 A } 330.50 & 0.825 \quad \text { II.2 } & 615\end{array}$

6082. $\Sigma$ 1615. I $2^{\mathrm{h}} 9^{\mathrm{m}} \cdot 6+33^{\circ} 17^{\prime}$. r9I2.249 I $\quad 87.50 \quad 27.03 .3 \quad$ I $2.2 \quad 308$ $\begin{array}{llllll}1912.249 & \mathrm{~A} & 88.06 & 26.903 & 12.3 & 308\end{array}$ \begin{tabular}{l}
$1912.307 . A$ \\
\hline
\end{tabular} 1912.249 L 87.5027 .033 I912.278 A $87.27 \quad 26.982$

6187. $\sum 1647 . \quad 12^{\mathrm{h}} 26^{\mathrm{m}} \mathrm{o}+10^{\circ} 13^{\prime}$.

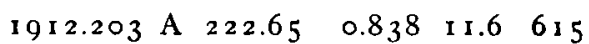

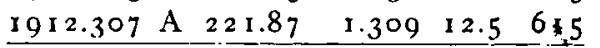
1912.255 A $222.26 \quad 1.074$
6243. $\Sigma$ 1670. $\gamma$ Virginis. I $2^{\mathrm{h}} 37^{\mathrm{m}} \cdot \mathrm{I}-0^{\circ} 57^{\prime}$.

I 9 I 2.285 I. $325^{\circ} .80 \quad 5.498 \quad$ I $2^{\mathrm{h}} \quad 378$ $\begin{array}{lllll}1912.285 \mathrm{~A} \quad 324.20 & 5.524 & 11.8 & 378\end{array}$ $1912.277 \mathrm{~L} \quad 326.10 \quad 5.459 \quad 14 \quad 378$ 19 I2.28 I L $325.95 \quad 5.478$ I9I2.285 A $324.20 \quad 5.524$

6212. $\Sigma_{1657 .} 24$ Comae. $12^{\mathrm{h}} 30^{\mathrm{m}} \cdot 6+18^{\circ} 5^{2^{\prime}}$.

$\begin{array}{llllll}1912.249 & \mathrm{~L} & 270.28 & 20.370 & 12.0 & 308\end{array}$ $\begin{array}{lllllll}1912.249 & A & 270.13 & 20.252 & 12.1 & 308\end{array}$

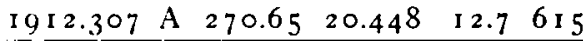

I 9 I 2.249 I. $270.28 \quad 20.370$

I912.278 A $270.3920 .35^{\circ}$

Der Hauptstern ist rotgelb, der Begleiter ist schwach grün.

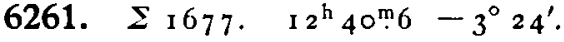

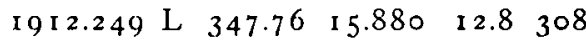
I9I 2.249 A 347.58 16.128 I 2.5308 I9I2.307 A 348.10 I6.II5 I3.0 308 1912.249 I, $347.76 \quad 15.880$ I9I2.278 A 347.84 I6.122

6390. ¿ I7rg. $13^{\mathrm{h}} 2^{\mathrm{m}} 7+\mathrm{I}^{\circ} 4^{\prime}$

$\begin{array}{llllll}\text { I } 912.249 & \mathrm{~L} & 359.20 & 7.108 & \text { I } 2.8 & 308\end{array}$ $\begin{array}{llllll}19 I 2.249 \mathrm{~A} & 0.10 & 7.23 \mathrm{I} & 13.0308\end{array}$ $1912.307 \mathrm{~A} \quad 0.65 \quad 7.253 \quad 13.4 \quad 308$ I912.249 I, $359.20 \quad 7.108$ $1912.278 \mathrm{~A} \quad 0.38 \quad 7.242$

6403. H 2644. $13^{\mathrm{h}} 3^{\mathrm{m} \cdot 7}+76^{\circ} 47^{\prime}$. $\begin{array}{lllll}\text { I9 I1.693 A } 253.85 & 39.666 & 0.5 & 209\end{array}$ $\begin{array}{llllll}\text { I } 11.704 & A & 252.75 & 39.142 & 23.8 & 209\end{array}$ 19 II.699 A $253.30 \quad 39.404$

6472. H 530. I $3^{\mathrm{h}} 18^{\mathrm{m}} \mathrm{G}+36^{\circ} 24^{\prime}$. $1911.320 \mathrm{~A} \quad 29.75 \quad 35.045 \quad 12.9 \times 67$ $9^{\mathrm{m}} \cdot 5, \quad 10^{\mathrm{m}}$.

6482. $\Sigma 1744$. \$ Ursae maj. $13^{\mathrm{h}} 20^{\mathrm{m}} \cdot 6+55^{\mathrm{o}} 24^{\mathrm{\prime}}$.

$\begin{array}{llllll}\text { I } 911.685 & \text { I, } & 150.2 & 14.574 & 2 \text { I } & 308\end{array}$ 6536. У 1760 . $13^{\mathrm{h}} 30^{\mathrm{m}} \cdot 3+26^{\circ} 44^{\prime}$. I9 12.3 I 5 L, $63.32 \quad 8.444$ I3 308

6545. H 228. I $3^{\mathrm{h}} 3^{\mathrm{Im}} \mathrm{I}+10^{\circ} 39^{\prime}$. $1911.320 \mathrm{~A} \quad \mathrm{I} 5.70-70.575 \quad 13.2 \quad 167$ $7^{\mathrm{m}}, 8 \mathrm{~m} \cdot 5$.

6783. $\Sigma_{1823} . \quad 14^{\mathrm{h}} 11^{\mathrm{m}} \cdot 5+10^{\circ} 43^{\prime}$. $\begin{array}{llllll}\text { I9I2.315 L } & 151.72 & 3.757 & 13.8 & 378\end{array}$ $8^{\mathrm{m}} \cdot 5,9^{\mathrm{m}} \cdot 5$

6837. $\Sigma_{18} 83$. I $4^{\mathrm{h}} 17^{\mathrm{m}} \cdot 8-7^{\circ} 4^{\prime}$.

$\begin{array}{llllll}\text { I } 912.3 \text { I } 5 \text { I, } 170.10 & 5.305 & 14.1 & 378\end{array}$
6887. $\Sigma_{1850} . \quad 14^{\mathrm{h}} 24^{\mathrm{m}} \cdot 8+28^{\circ} 4^{2}$. 1911.312 A 261 . 10 25:593 13\%.8 167 1911.334 A $262.62 \quad 25.803 \quad 13.8 \quad 167$ I 911.323 A 26 I.86 25.698

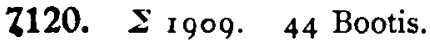
$15^{\mathrm{h}} \mathrm{I}^{\mathrm{m}} \cdot 4+48^{\circ} 0^{\prime}$.

$\begin{array}{lllll}\text { I9II.7OI L } & 24 \text { I.I } 5 \quad 3.993 \quad 22.2 & 469\end{array}$

7318. $\Sigma_{1954 .} \delta$ Serpentis. $15^{\mathrm{h}} 30^{\mathrm{m}} \cdot 6+10^{\circ} 5^{\circ}$.

$\begin{array}{llllll}\text { I9I } 2.277 & \mathrm{~L} & 180.3 & 3.378 & \text { I } 4.5 & 378\end{array}$ $1912.315 \mathrm{~L}$ 1 81.3 1912.296 L I $80.8 \quad 3.404$

7327. $\mathrm{H} 2788$. $15^{\mathrm{h}} 3^{2 \mathrm{~m}} \cdot \mathrm{o}+45^{\circ} \mathrm{I}^{\prime}$. $\begin{array}{lllll}\text { I911.70I A } 304.40 \quad 56.947 & 22.5 & \text { I67 }\end{array}$ $\begin{array}{lllll}1911.739 \text { A } 304.62 & 57.483 & 23.9 & 209\end{array}$ 1911.720 A 304.5157 .215

9789. H $145^{8}$. I $9^{\mathrm{h}} 55^{\mathrm{m}} \cdot 9+10^{\circ} 54^{\mathrm{\prime}}$. $\begin{array}{llllll}\text { I } 911.700 \mathrm{~A} & \mathrm{I} 33.55 & 16.534 & 22.3 & 308\end{array}$ I9II.739A I 31.92 I 6.52 I 0.5209 $1911.720 \mathrm{~A} 132.74 \quad 16.527$

9805. H I 462 . I $9^{\mathrm{h}} 5^{6^{\mathrm{m}}} \cdot 5+25^{\circ} 40^{\prime}$. $\begin{array}{llllll}1911.700 \mathrm{~A} & 25.05 & 32.545 & 21.7 & 167\end{array}$ I 911.739 A $\quad 24.90 \quad 33.238 \quad 0.9 \quad 209$ I911.720 A $24.98 \quad 32.892$

9945. H 606. $20^{\mathrm{h}} 5^{\mathrm{m}} \cdot 4+37^{\circ} 5^{\prime}$. 1911.700 A $228.2 \quad 43.776 \quad 22 \quad 167$

10085. $\Sigma_{2675 .} x$ Cephei. $20^{\mathrm{h}} 14^{\mathrm{m} \cdot \mathrm{O}} \mathrm{O}+77^{\circ} 26^{\mathrm{s}}$.

$\begin{array}{llllll}19 I 1.67 \text { I L I } 22.90 & 7.252 & 0.3 & 469\end{array}$ $\begin{array}{llllll}\text { I } 9 \text { I I.693 I, I } 21.03 & 7.135 & 0.8 & 308\end{array}$ $\begin{array}{lllllll}1911.693 \mathrm{~A} & 121.93 & 7.253 & \text { 1.0 } & 308\end{array}$ 1911.682 L, $121.97 \quad 7.194$ I9II.693 A I $21.93 \quad 7.253$

10235. $\mathrm{H}_{15} 16.20^{\mathrm{h}} 23^{\mathrm{m}} \cdot 9+54^{\circ} 13^{\prime}$. $\begin{array}{lllll}1911.693 \mathrm{I} & 11.70 \quad 13.708 \quad 2 & 308\end{array}$ $\begin{array}{llllll}1911.693 & \mathrm{~A} & 14.00 & \mathrm{r} 3.353 & 1.3 & 308\end{array}$

10350. H I 543. $20^{\mathrm{h}} 3 \mathrm{I}^{\mathrm{m}} 8+33^{\circ} 4^{\prime}$. $\begin{array}{lllll}1911.700 \mathrm{~A} & 26.88 & 16.141 & 20.7 & 167\end{array}$

10361. I $2703.20^{\mathrm{h}} 32^{\mathrm{m}} \cdot 7+\mathrm{I} 4^{\circ} 25^{\prime}$. $\mathrm{AB}$.

1911.676 L $290.47 \quad 25.475 \quad 22.0 \quad 308$ $\mathrm{AC}$.

$\begin{array}{llllll}1911.676 \quad L & 236.70 & 71.818 & 22.2 & 308\end{array}$

10623. $2273^{6} .20^{\mathrm{h}} 5^{2^{\mathrm{m}} \cdot 5}+\mathrm{I} 2^{\circ} 39^{\prime}$. $\begin{array}{llllll}1911.701 & \text { I. } & 217.65 & 4.949 & 23.1 & 308\end{array}$ 
10725. $\Sigma 2754 . \quad 21^{\mathrm{h}} 2^{\mathrm{m}}$ 。 $+12^{\circ} 49^{\prime}$. $\begin{array}{lllllll}1911.687 & \mathrm{~L} & 300^{\circ} .35 & 32^{\text {. }} .635 & 21^{\text {h }} .9 & 308\end{array}$ \begin{tabular}{rlllll}
$1911.690 \mathrm{~L}$ & $300.50 \quad 33.000$ & 20.5 & 308 \\
\hline
\end{tabular} 1911.688 L $300.433^{2.817} 8^{\mathrm{m}}, 9^{\mathrm{m}}$

11272. $\Sigma_{2828} . \quad 2^{\mathrm{h}} 44^{\mathrm{m}} \cdot 9+2^{\circ} 5^{\prime}$. $\mathrm{AB}$.

$\begin{array}{llllll}19 I 1.687 & \mathrm{~L} & 142.50 & 28.709 & 2 \mathrm{I} & 308\end{array}$ $\mathrm{BC}$.

$\begin{array}{llllll}1911.687 & \mathrm{~L} & 37.75 & 3.364 & 21.3 & 469\end{array}$

11335. $\Sigma_{2841 .} 21^{\mathrm{h}} 50^{\mathrm{m}} \cdot 2+19^{\circ} 27^{\prime}$. $\begin{array}{llllll}\text { I9II.676 L II } 0.50 & 22.398 & 21.3 & 308\end{array}$ $\begin{array}{llllll}1911.689 \mathrm{~L} & \text { IIr.10 } & 22.152 & 22.0 & 308\end{array}$ $\begin{array}{llllll}1911.689 & \mathrm{~A} & 110.45 & 22.046 & 22.4 & 308\end{array}$ I9I1.683 L $110.8022 .275 \quad 6^{\mathrm{m}} \cdot 5,8^{\mathrm{m}}$ I9II.689A II0.45 22.046

11481. $2_{2857 . ~} 2^{\mathrm{h}} \mathrm{I}^{\mathrm{m}} 8+9^{\circ} 40^{\prime}$. $\begin{array}{llllll}1911.646 \text { L II } 3.98 & \text { I } 9.7 \text { I } 5 & 22.8 & 295\end{array}$ $\begin{array}{llllll}1911.663 \mathrm{~L} & \text { I } 13.70 & \text { I } 9.516 & 22.5 & 469\end{array}$ 19 II.655 L II 3.84 I9.6I6

$7^{\mathrm{m}}$ weiß, $9^{\mathrm{m}}$ blau.

11486. $\Sigma 2861 . \quad 22^{\mathrm{h}} \mathrm{I}^{\mathrm{m}} \cdot 9+20^{\circ} 22^{\prime}$. $\begin{array}{llllll}1911.689 & L & 219.80 & 7.043 & 22.8 & 308\end{array}$ $\begin{array}{llllll}\text { I9II.7OI L } & 2 \text { I } 9.65 & 7.318 & 23.7 & 308\end{array}$ I9I1.695 L 2 I9.73 7.18 I $7^{\mathrm{m}} \cdot 3,8^{\mathrm{m}} \mathrm{I}$
11667. $\Sigma_{2897 .} 22^{\mathrm{h}}{ }_{1} 7^{\mathrm{m}} \cdot 5+\mathrm{I} 4^{\circ} 28^{\prime}$.

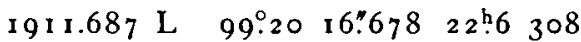
$\begin{array}{lllllll}911.690 & \mathrm{I} & 98.90 & 16.586 & 22.1 & 308\end{array}$ I9Ir.688 L $99.05 \quad 16.632$ $\begin{array}{llllll}\text { IgIr.67I A } 100.60 \quad 17.044 & 22.6 & 308\end{array}$ $8^{\mathrm{m}} \cdot 7,9^{\mathrm{m}} \cdot 5$.

11743. $\Sigma 2909 . \zeta$ Aquarii. $22^{\mathrm{h}} 24^{\mathrm{m}} \cdot 2-0^{\circ} 29^{\prime}$.

19II.646 L $314.32 \quad 3.155 \quad 22.5 \quad 469$ $\begin{array}{llllll}\text { I9II.663 L } & 311.65 & 2.940 & 22.3 & 469\end{array}$ I9II.655 L 3 I 2.98 $3.047 \quad 4^{\mathrm{m}}, 4^{\mathrm{m}}$

11789. $\Sigma_{2915 .} 22^{\mathrm{h}} 28^{\mathrm{m}} \mathrm{r}+6^{\circ} 57^{\prime}$. $\begin{array}{lllllll}\text { I } 911.632 & \text { I. I } 46.40 & \text { I } 2.660 & 21.0 & 302\end{array}$ \begin{tabular}{l}
$1911.639 \mathrm{~L} \quad \mathrm{I} 46.05 \quad 12.567$ \\
\hline
\end{tabular}

I 9 I I.6.36 L I 46.23 I 2.6 I 3

$8^{\mathrm{m}} \cdot 5$ weiß, $8^{\mathrm{m}} .8$ weiß.

11814. \2920. $22^{\mathrm{h}} 30^{\mathrm{m}} \cdot \mathrm{o}+3^{\circ} 45^{\prime}$. $\begin{array}{lllllll}\text { I } 911.693 & \mathrm{~L} & 145.95 & 13.746 & 0.0 & 308\end{array}$ $\begin{array}{lllllll}1911.742 & \mathrm{~L} & 145.55 & \text { I 3.II } 8 & 22.0 & 308\end{array}$ I9II.7I8 I, I $45.75 \quad 13.432$

11818. $\mathrm{H}_{17} 85.22^{\mathrm{h}} 30^{\mathrm{m}} \cdot 6+29^{\circ} 15^{\prime}$. $\begin{array}{llllll}\text { I } 9 \text { II.693 } \mathrm{A} & \text { I } 7 \text { I.83 } & \text { I } 3.942 & 23 & 308\end{array}$

11850. $229^{2} 5 . \quad 22^{\mathrm{h}} 33^{\mathrm{m}} \cdot 3+5^{\circ} 26^{\prime}$.
12222. H $3176.23^{\mathrm{h}} 8^{\mathrm{m}} \cdot 4+\mathrm{r}^{\circ} 3^{\prime}$. 1911.700 A I65.05 $26^{\prime \prime} .77$ I $22^{\mathrm{h}} .9308$

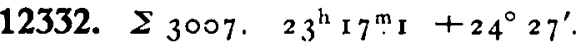
$\begin{array}{llllll}\text { I } 9 \text { II.742 I, } & 84.17 & 5.328 & 23.7 & 469\end{array}$ $\begin{array}{lllll}19 \text { II.787 I, } & 81.50 & 5.721 & 23.5 & 469\end{array}$ I9II.764 I, $82.84 \quad 5.524$

12406. 2 3019. $23^{\mathrm{h}} 26^{\mathrm{m}} \mathrm{I}+4^{\circ} 45^{\prime}$.

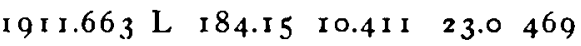
I 9 I I.687 L I $84.80 \quad$ I0.892 $23.1 \quad 308$ I 9 1 $1.675 \mathrm{~L}$ 184.48 10.6 I I $^{\mathrm{m} \cdot 2,} 7^{\mathrm{m}} .9$

- Nur 3 Einstellungen. Ein Wolkenschleier unterbrach die Beobachtung.

I)asselbe Paar.

$\begin{array}{llllll}\text { I } 911.67 \text { I A } & 185.12 & 10.905 & 23.3 & 308\end{array}$

12538. H 32 II. $23^{\mathrm{h}} 40^{\mathrm{m}} \cdot 3+3^{\circ} 6^{\prime}$. $\begin{array}{llllll}\text { I9II.704 A } & 82.87 & 48.475 & 22.8 & 167\end{array}$

12567. $23040.23^{\mathrm{h}} 43^{\mathrm{m}} \cdot 5+9^{\circ} 39^{\prime}$.

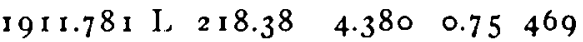

12675. $23050.23^{\mathrm{h}} 54^{\mathrm{m}} \cdot 9+33^{\circ} 14^{\prime}$. $\begin{array}{lllll}1911.663 \mathrm{~L} & 217.15 & 2.513 & 23.5 & 703\end{array}$ $\begin{array}{llllll}1911.687 & I & 218.02 & 2.369 & 23.6 & 703\end{array}$ I911.67I A $220.10 \quad 2.461 \quad 23.6 \quad 703$ I911.675 L $217.592 .44 \mathrm{I} \quad 6^{\mathrm{m}}, 6^{\mathrm{m}}$ I9I1.67IA $220.102 .46 \mathrm{r}$

Urania-Sternwarte, Kopenhagen, I 9 I 2 Juni.

C. Luplau-Fanssen, E. Buch-Andersen.

\section{Sur la nouvelle comète 1912 b (Schaumasse). Par MM. Fayet et Schaumasse.}

A l'aide des observations obtenues les i 8 , i 9 et 20 Oct. par M. Schaumasse, avec notre équatorial coudé, nous avons calculé, M. Schaumasse et moi, une parabole provisoire, dont voici les éléments:

$$
\begin{aligned}
& T=\text { I9I2 Oct. } 25.3 \text { I } 53 \text { t.m. Paris } \\
& \pi=113^{\circ} 50^{\prime} 20^{\prime \prime} \\
& \left.\delta=270235^{8}\right\} 1912.0 \\
& i=535234 \\
& \log q=0.021444
\end{aligned}
$$

Représentation du lieu moyen: $O-C\left\{\begin{aligned} \cos \beta \mathrm{d} \lambda & =-4.0 \\ \mathrm{~d} \beta & =-0.8 \text {. }\end{aligned}\right.$

On constate immédiatement l'analogie frappante que présentent ces éléments (exception faite de $T$ ) avec ceux de la comète Tuttle, que nous donnons ci-dessous

$$
\begin{aligned}
& T=\text { 1913 Janvier } 4 \\
& \pi=116^{\circ} 39^{\prime} \\
& \delta=270001912.0 \\
& i=5429 \\
& \log q=0.00823
\end{aligned}
$$

Mais les dates relatives au passage au périhelie sont très différentes; d'autre part, les déclinaisons géocentriques ne se ressemblent nullement.
En effet, alors que, le 20 Octobre, la nouvelle comète se trouvait par: $\alpha=10^{\mathrm{h}} 3^{\mathrm{m}}, \delta=-0^{\circ} 43^{\prime}$, l'éphéméride publiée récemment par M. Miliéević (A. N. 4602) indique pour la comète Tuttle à la même date, les coordonnées suivantes: $\alpha=9^{\mathrm{h}} 37^{\mathrm{m}}, \delta=+73^{\circ} 27^{\prime}$.

Néanmoins, étant donné la ressemblance offerte par les autres éléments, j'ai cherché, s'il ne serait pas possible de représenter une observation du nouvel astre (celle du ig Octobre) à l'aide des éléments de la comète Tuttle, en modifiant d'une façon appropriée, l'élément $T$.

Parmi les divers essais effectués, voici celui (A) qui conduit à la meilleure représentation et ceux (B et $C$ ) qui satisfont rigoureusement à une seule des coordonnées.

$$
\begin{aligned}
& \text { Essai } \\
& \text {, } \\
& \text { (A) } \\
& \text { - } \mathrm{II}^{\circ} 7^{\prime} \\
& \text { (B) } \\
& \text { - } 12^{\circ} 4^{\prime} \\
& \text { Oct. } 28.3^{2} \\
& \text { Oct. } 29.07 \\
& -26.4 \\
& \begin{array}{r}
0^{\circ} \\
-55.3
\end{array}
\end{aligned}
$$

Il n'est donc pas douteux, sinon que la nouvelle comète est identique avec la comète Tuttle, du moins qu'elle se meut dans une orbite presque semblable.

L'identité impliquerait une correction de plus de 2 mois pour l'époque du passage au périhélie conclue des éléments 